EPJ Web of Conferences 28, 12006 (2012)

DOI: $10.1051 /$ epjconf/20122812006

(c) Owned by the authors, published by EDP Sciences, 2012

\title{
Triggering On Hadronic Tau Decays: A challenge met by ATLAS
}

\author{
Marcus M. Morgenstern On behalf of the ATLAS Collaboration
}

Technical University Dresden, Dresden, Germany

\begin{abstract}
The ATLAS experiment at the Large Hadron Collider (LHC) has been able to collect $5.25 \mathrm{fb}^{-1}$ of data in 2011. For many physics analyses both in context of the Standard Model (SM) and Beyond the Standard Model (BSM) theories such as Higgs boson searches, tau leptons play an important role. Thus, triggering on hadronic tau decays is an essential ingredient for the success of those measurements. This contribution will summarize the developed efforts to meet this challenge. Efficiency measurements using data taken in 2011 at a center-of-mass energy of $7 \mathrm{TeV}$ are described and results are presented. An outlook on further developments of the tau trigger algorithms, to match future requirements and higher instantaneous luminosities are summarised in the end.
\end{abstract}

\section{Introduction}

Tau leptons play a crucial role in many physics processes investigated by the ATLAS experiment at the Large Hadron Collider (LHC), both within the Standard Model and in models beyond the SM. In particular for Higgs boson decays in the SM at low masses and in the Minimal Supersymmetric extension of the SM (MSSM) the branching fraction to taus is expected to be in the order of $10 \%$. For this purpose it is important to trigger on tau decays efficiently. The tau trigger is aimed at efficiently rejecting background events with fake tau candidates while keeping the acceptance for real taus as high as possible. Tau leptons decay hadronically $65 \%$ of the time and leptonically $35 \%$ of the time. Only hadronic tau decays are considered in this context. Taus tend to decay into one or three charged hadrons, mainly pions and to a small amount into kaons, plus additional neutral pions or kaons. Thus, the tau decay is characterised by one or three charged tracks and narrow energy deposists in the electromagnetic and hadronic calorimeter. These decay patterns become manifest in isolation criteria applied to distinguish tau decays from background, mainly arising from QCD multijet contributions.

\section{The ATLAS Tau Trigger}

The ATLAS trigger system [1] is aimed at reducing the initial collision rate of $40 \mathrm{MHz}$ to a reliable data rate for permanent storage of around $200-400 \mathrm{~Hz}$. This is achivied by a three-level system. The first system is the level 1 (L1) trigger system. It is a hardware based system which uses information from the electromagnetic (EM) and hadronic calorimeter systems, provided as trigger towers of size $\Delta \eta \times$ $\Delta \phi=0.1 \times 0.1$. At level 1 a tau lepton is identified by applying a given transverse energy, $E_{\mathrm{T}}$, cut corresponding to the given trigger item, e.g. for L1_tau15 a $E_{\mathrm{T}}$ threshold strictly above $15 \mathrm{GeV}$ is applied. The energy is reconstructed by the sum of energy deposits in the highest $2 \times 1$ pair of EM towers and the sum of energy deposits of $2 \times 2$ hadronic towers behind the EM layers. In addition to the $E_{\mathrm{T}}$ threshold a cut on the energy in the isolation region can be applied which is measured as the sum of energy deposits in an isolation region in a ring of $4 \times 4$ around the core region of $2 \times 2$ towers. A so-called Region of Interest (RoI) is built during the L1 processing and is passed to the Higher Level Trigger (HLT), if the event is accepted. To stay below the rate limit of $75 \mathrm{kHz}$ a hardware based pre-scale (PS) factor can be applied. Next, the level 2 (L2) system performs track reconstruction within the RoI in addition to calorimeter information (using the full detector granularity in the RoI) to identify tau leptons. Identification variables measuring tau decay properties, such as the narrowness of the tau decay products or the number of reconstructed tracks, are used to provide a sufficient discrimination power against QCD jets. By setting requirements on those identification variables, several working points corresponding to given signal efficiencies are defined. In addition a PS can be applied. Figure 1 presents the electromagnetic radius, $R_{\mathrm{EM}}$, which measures the lateral shape of the tau decay. Figure 2 shows the number of tracks for L2 taus obtained in signal Monte Carlo (MC), and background QCD di-jet data.

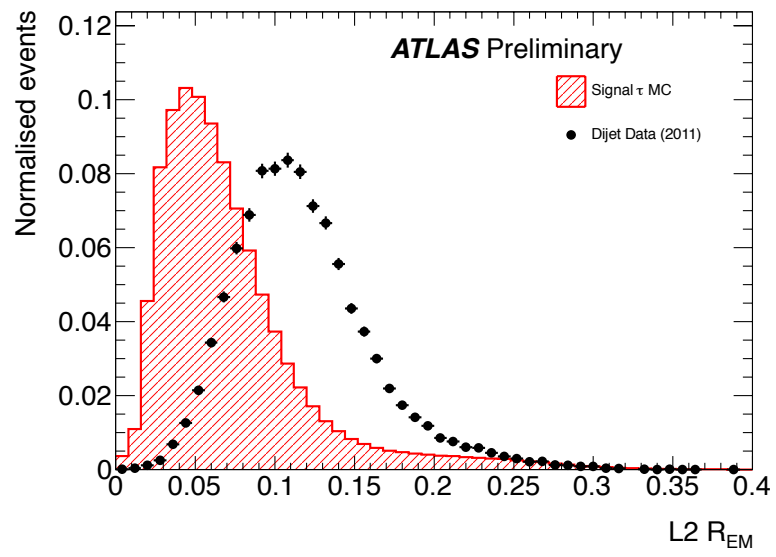

Fig. 1. Electromagnetic radius, $R_{e m}$, for trigger taus in signal Monte Carlo and QCD di-jet data. The latter serves as background estimate [2]. 


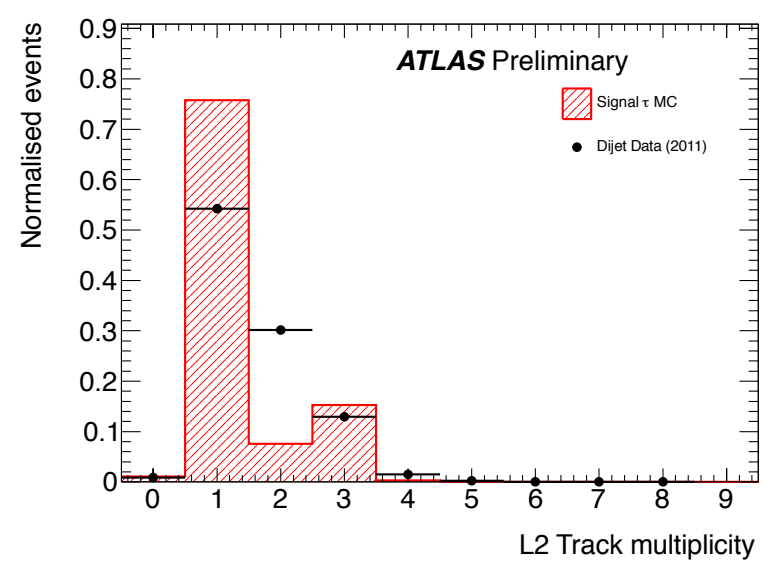

Fig. 2. Number of tracks of L2 taus for trigger taus in signal Monte Carlo and QCD di-jet data. The latter serves as background estimate [2].

The last trigger step is accomplished by the event filter (EF), which runs on fully built events.. Reconstruction and identification algorithms similar to the ones used for offline analysis are utilised [3]. Depending on the trigger chain a PS factor is applied to match the final ouput rate of around $200-400 \mathrm{~Hz}$.

\section{Tau Trigger Efficiency}

For physics analyses it is mandatory to know the trigger efficiency. This is the probability for an offline-reconstructed and identified tau to pass the trigger. For this purpose several studies measuring the trigger efficiency have been performed using both Monte Carlo simulated and real data events.

To measure the tau trigger efficiency a study based on $Z \rightarrow \tau \tau$ MC events has been performed. The efficiency is measured for several trigger items as a function of the transverse energy, $E_{\mathrm{T}}$. Since MC simulation will not necessarily give a reasonable description of the new energy frontier of the LHC collisions, data driven measurements of the trigger efficiency are essential. Hence, a study using $Z$ boson decays in real data has been performed. Herein the trigger efficiency is measured by a tag-and-probe method using data of the 2011 run at a center of mass energy of $\sqrt{s}=7 \mathrm{TeV}$. An event is tagged by either an electron or a muon, by applying an unbiased electron or muon trigger, respectively. On the probe side tau leptons which are reconstructed and identified by the offline algorithms are used to measure the tau trigger efficiency. The amount of data analysed in this measurement corresponds to roughly $475 \mathrm{pb}^{-1}$. The results for the EF tau_20_medium and tau_29_medium items are shown in Figure 3 and 4. For these items medium tau trigger identification is applied as well as a cut on $E_{\mathrm{T}}$ of $20 \mathrm{GeV}$ and $29 \mathrm{GeV}$, respectively.

To gain knowledge of the tau trigger efficiencies at very high energies, where a SM sample of real tau leptons is limited in a few $\mathrm{pb}^{-1}$ of data, QCD di-jet events can be used to achieve a reasonable precision. Such a measurement is based on the idea that a jet faking a reconstructed and identified offline tau lepton should fire the tau trigger at high $p_{\mathrm{T}}$ at the same rate as real tau leptons. Hence, a

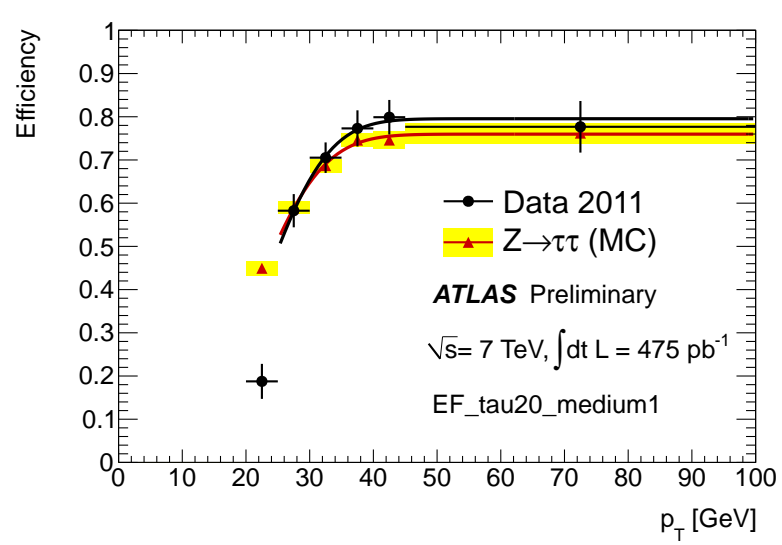

Fig. 3. Tau trigger efficiency measured in $Z \rightarrow \tau \tau$ decays using 2011 data by applying a tag-and-probe method for the EF tau_20_medium item. The efficieny is measured w.r.t. offline tau candidates passing medium identification requirements as a function of the offline tau $p_{\mathrm{T}}[2]$.

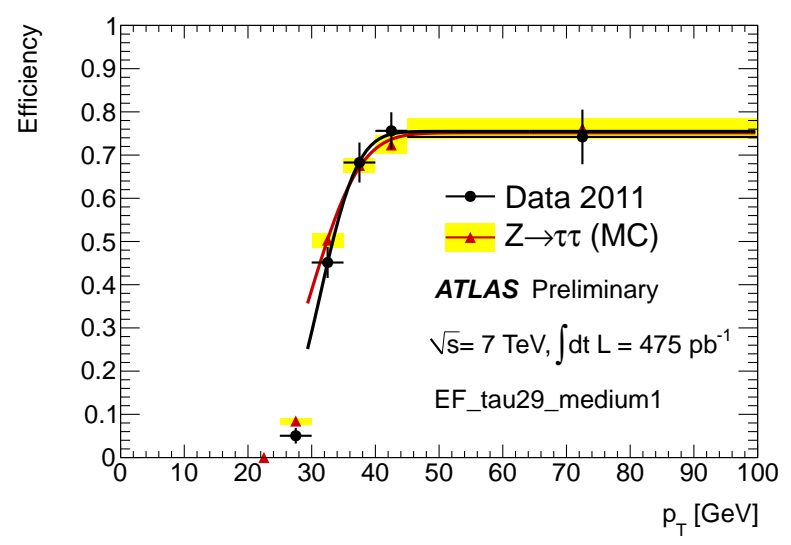

Fig. 4. Tau trigger efficiency measured in $Z \rightarrow \tau \tau$ decays using 2011 data by applying a tag-and-probe method for the EF tau_29_medium. The efficieny is measured w.r.t. offline tau candidates passing medium identification requirements as a function of the offline tau $p_{\mathrm{T}}[2]$.

di-jet sample is selected in collision data and the trigger efficiency is measured by a tag-and-probe method.

\section{Future prospects for 2012 data}

After a maintance stop in early 2012 the LHC will restart proton-proton collisions in early April. To deal with the expected increase in instantaneous luminosity, and thus with a larger pile-up contribution as well as higher trigger rates, improvements in the selection algorithms of the tau trigger have to be implemented. The evolution of the event rate as a function of the instantaneous luminosity is shown in Figure 5 and 6 for different L1 and EF items, respectively.

Several options are available to match the given fixed output rate. The straightforward way is to increase the $E_{\mathrm{T}}$ of the items without prescale. Furthermore, the isolation criteria can be tightened. Both result in a lower acceptance for physics analysis. A different approach to obey the necessary rejection rate is to apply more advanced algorithms, as used in offline identification. Due to the higher rejection power at a given signal efficiency working point, mulitvariate techniques could be a reasonable alternative to the 


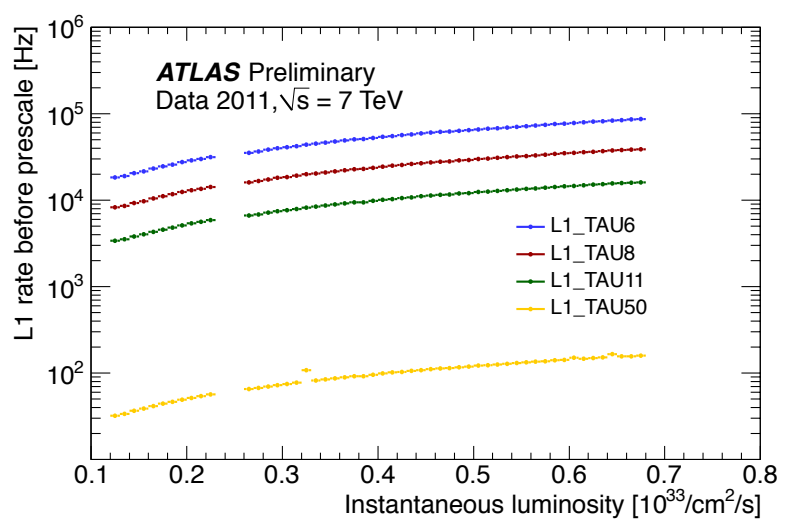

Fig. 5. Evolution of the event rate as a function of the instantaneous luminosity for L1 items as measured in 2011 data [2].

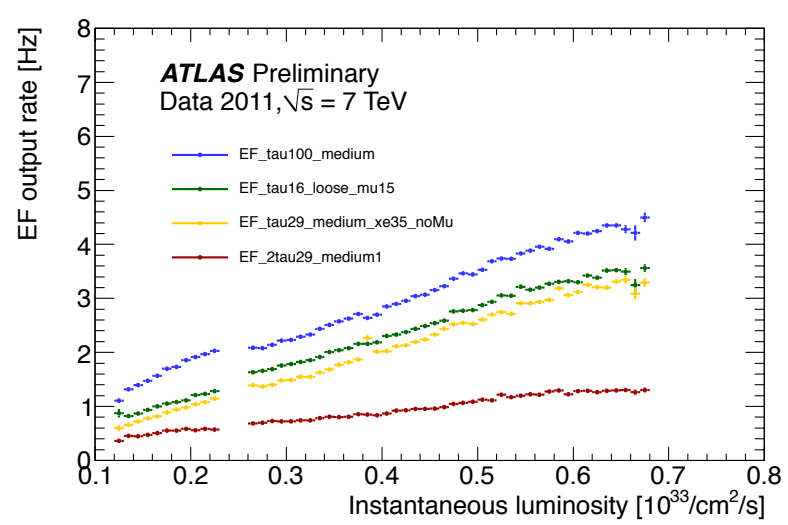

Fig. 6. Evolution of the event rate as a function of the instantaneous luminosity for EF items as measured in 2011 data [2].

current cut based implementation of the tau trigger algorithms. This is planned for the 2012 data taking.

\section{References}

1. ATLAS Collaboration, The Expected Performance of the ATLAS Experiment - Detector, Trigger and Physics, CERN-OPEN-2008-020, https://cdsweb.cern.ch/record/1125884

2. https://twiki.cern.ch/twiki/bin/view/AtlasPublic/TauTriggerPublicResults

3. ATLAS Collaboration, Reconstruction, Energy Calibration, and Identification of Hadronically Decaying Tau Leptons in the ATLAS Experiment, ATLAS-CONF-2011077, https://cdsweb.cern.ch/record/1353226 